\title{
Erratum to: Modelling and experimental study of the tertiary creep stage of Grade 91 steel
}

\author{
Rattanak Lim • Maxime Sauzay • France Dalle • \\ Ivan Tournie - Patrick Bonnaillie • \\ Anne-Françoise Gourgues-Lorenzon
}

Published online: 29 March 2011

(C) Springer Science+Business Media B.V. 2011

\section{Erratum to: Int J Fract DOI 10.1007/s10704-011-9585-y}

Due to an unfortunate turn of events this article has been published with an erroneous version of Table 5 . Please find on this page the correct Table 5 that should be regarded by the reader as the final version.
Table 5 Norton law parameters

\begin{tabular}{lrl}
\hline Temperature $\left({ }^{\circ} \mathrm{C}\right)$ & $N$ & $C\left(\mathrm{MPa}^{-N} \mathrm{~h}^{-1}\right)$ \\
\hline 500 & 20.35 & $1.56 \times 10^{-55}$ \\
550 & 16.76 & $6.97 \times 10^{-45}$ \\
600 & 9.94 & $8.42 \times 10^{-27}$ \\
\hline
\end{tabular}

The online version of the original article can be found under doi:10.1007/s10704-011-9585-y.

R. Lim $(\varangle) \cdot$ M. Sauzay $\cdot$ F. Dalle $\cdot$ I. Tournie DEN/DMN/SRMA, CEA Saclay,

91191 Gif-sur-Yvette, France

e-mail: rattanak.lim@cea.fr

P. Bonnaillie

DEN/DMN/SRMP, CEA Saclay,

91191 Gif-sur-Yvette, France

R. Lim · A.-F. Gourgues-Lorenzon

Centre des Matériaux UMR CNRS 7633, MINES ParisTech, BP 87, 91003 Evry, France 